Recepción: 19 / 11 / 2018

Aceptación: 28 / 12 / 2018

Publicación: 20 / 02 / 2019

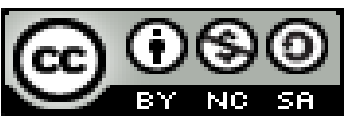

Ciencias de la salud

Artículo de Investigación

\title{
Nivel socioeconómico y cultural de los estudiantes de la carrera de odontología de la Universidad Nacional de Loja y su relación con el estado de salud buco-dental
}

\section{Socioeconomic and cultural level of the students of the career of dentistry of the National University of Loja and its relationship with the state of dental-dental health}

\section{Nível socioeconômico e cultural dos discentes da carreira de odontologia da Universidade Nacional de Loja e sua relação com o estado de saúde odontológica}

\author{
Deisy P. Saraguro-Ortega ${ }^{\mathrm{I}}$ \\ deisy.saraguro@unl.edu.ec \\ Diego R. Aguilar-Ochoa II \\ diego_rene1993@hotmail.com \\ Jhoanna A. Riofrio-Herrera ${ }^{\text {III }}$ \\ jhoanna.riofrio@unl.edu.ec
}

Tannya L. Valarezo-Bravo IV

tannya.valarezo@unl.edu.ec

Ana M. Granda-Loaiza V

ana.granda@unl.edu.ec

Correspondencia: deisy.saraguro@unl.edu.ec

\footnotetext{
I. Magister en Salud Publica con Enfasis en Gestion; Diploma Superior en Odontologia Restauradora y Estetica; Diploma Superior en Manejo de Pacientes en Odontopediatria; Doctor en Odontologia; Universidad Nacional de Loja, Loja, Ecuador.

II. Odontologo de la Universidad Nacional de Loja, Loja, Ecuador.

III. Especialista en Rehabilitacion Oral; Odontologa; Universidad Nacional de Loja, Loja, Ecuador.

IV. Especialista en Odontopediatria; Odontologa; Universidad Nacional de Loja, Loja, Ecuador.

v. Especialista en Ortodoncia; Diploma Superior en Odontologia Restauradora y Estetica; Doctora en Odontologia; 
Deisy P. Saraguro-Ortega; Diego R. Aguilar-Ochoa; Jhoanna A. Riofrio-Herrera; Tannya L. Valarezo-Bravo; Ana M.

\title{
Resumen
}

Uno de los grandes problemas que afecta a la población mundial es la crisis económica, vivir en condiciones de pobreza y limitados en cuanto al acceso a los servicios de salud siendo uno de los principales factores de riesgo. Se realizó un estudio observacional, descriptivo y transversal a los estudiantes de la carrera de odontología de la universidad nacional de Loja siendo la población de estudio de 230 estudiantes con la finalidad de identificar el nivel socioeconómico y cultural de los estudiantes y su relación con el estado de salud buco-dental,. En la serie se obtuvo que las patologías de mayor frecuencia encontradas fueron caries dental, enfermedad periodontal, mal posición dentaria y hábitos para funcionales: de las cuales la caries dental, enfermedad periodontal y la mal posición dentaria tienen relación directa con la situación socioeconómica y cultural de los estudiantes, con un nivel de significancia de 0,0 de la prueba estadística Chi cuadrado de Pearson. En cuanto a los hábitos para funcionales se determinó un nivel de significancia de 0,4 lo que nos indica que no influye en estas, el nivel socioeconómico y cultural del grupo de estudio.

Palabras claves: Nivel Socioeconómico y Cultural; Salud Bucodental.

\begin{abstract}
One of the great problems that affects the world population is the economic crisis, living in conditions of poverty and limited in terms of access to health services being one of the main risk factors. An observational, descriptive and cross-sectional study was conducted to the students of the dentistry career of the National University of Loja. The study population was 230 students with the purpose of identifying the socioeconomic and cultural level of the students and their relationship with the state. of dental-dental health. In the series it was found that the most frequent pathologies found were dental caries, periodontal disease, poor dental position and functional habits: of which dental caries, periodontal disease and poor dental position are directly related to the socioeconomic and cultural situation of the students, with a significance level of 0.0 of the Pearson Chi square test. Regarding functional habits, a level of significance of 0.4 was determined, which indicates that the socioeconomic and cultural level of the study group does not influence these.
\end{abstract}

Keys words: Socioeconomic and Cultural Level; Oral Health 
Nivel socioeconómico y cultural de los estudiantes de la carrera de odontología de la Universidad Nacional de Loja y su relación con el estado de salud buco-dental

\section{Resumo.}

Em 2005, a OMS definiu, após o Workshop sobre "Otimização de cuidados", a designação de "prematuros prematuros" aqueles nascidos entre 34 semanas 0 dias e 36 semanas 7 dias após o início do último período menstrual. A imaturidade das funções do órgão, complicações e distúrbios específicos da prematuridade determinam a suscetibilidade a uma ampla variedade de doenças que diferem em comparação com os recém-nascidos normais. Sabendo que a prematuridade e o baixo peso ao nascer são um problema para o mundo e que tendem a aumentar, apesar das ações preventivas de saúde e dos avanços no cuidado perinatológico intensivo. Neste caso, pretendemos não apenas identificar os motivos que contribuem para o nascimento inerente ao período gestacional, mas também delinear quais seriam as conseqüências para e para o recém-nascido prestar o cuidado e a atenção necessários para preservar a vida da criança. novo ser. Pretende-se fazer uma revisão bibliográfica que sintetize a definição do recém-nascido, então avalie a influência do baixo peso na vida do recém-nascido. A primeira coisa que a metodologia qualitativa nos mostra é que o critério do pesquisador contribui no destino do resultado final. Embora o prognóstico e a sobrevida dos prematuros estejam diretamente relacionados à idade gestacional e ao peso ao nascer, é importante que os profissionais de saúde considerem a implementação de programas de acompanhamento como objeto de pesquisa para o recém-nascido prematuro e liberado. , a fim de verificar os efeitos benéficos de intervenções precoces em neonatos, os resultados podem ajudar a avaliar intervenções e melhorar o atendimento.

Palavras chaves: Nível Socioeconômico e Cultural; Saúde Oral.

\section{Introducción.}

La salud bucal es parte integrante de la salud general, pues un individuo no puede considerarse completamente sano si existe presencia activa de enfermedad bucal. Las enfermedades bucales, en particular la caries dental, las periodontopatías y las mal oclusiones, han sido subvaloradas por no ocasionar mortalidad directa, cuando en realidad su elevada frecuencia, molestias locales, estéticas y la repercusión en la salud general que ocasionan, justifica plenamente su atención como problema de salud pública. (Zacca, Sosa, \& Mojaiber, 2001) 
Deisy P. Saraguro-Ortega; Diego R. Aguilar-Ochoa; Jhoanna A. Riofrio-Herrera; Tannya L. Valarezo-Bravo; Ana M.

En el orden epidemiológico las enfermedades bucales, las más frecuentes en todas las poblaciones son la caries dental y las periodontopatías, que solo varían en su gravedad, prevalencia, y también entre diversos grupos de edades, y es más alta en las poblaciones con situación económica desventajosa.

Diferentes autores se han referido a la relación de la salud oral con el nivel socioeconómico. Su influencia es inversamente proporcional y, aunque los mecanismos por los que repercute son numerosos, podemos resumirlos en dos aspectos: los más pobres tienen menor nivel de educación, en relación con hábitos saludables, y tienen mayores necesidades que, a su vez, se satisfacen con menor frecuencia. A lo largo del tiempo se ha observado que, a diferencia de lo que ocurre con otras consultas médicas, las consultas al dentista son más frecuentes en individuos con estudios superiores y en los grupos socioeconómicos altos. (Bravo, Casals, Cortes, \& Llodra, 2006), (Petersen, Bourgeois, Ogawa, Estupinan-Day, \& Ndiaye, 2005), (Cuenca , 2005, págs. 263-74)

Basado en la problemática a nivel general y por la escasa información acerca del tema, se propuso realizar este estudio con la finalidad de conocer estado de salud bucodental actual de los estudiantes de la carrera de Odontología de la Universidad Nacional de Loja y relacionarlo con la situación socioeconómica y cultural de los mismos, a fin de determinar acciones de promoción y prevención de las patologías bucodentales.

\section{Material y método.}

Se realizó un estudio descriptivo y transversal con la finalidad de identificar el nivel socioeconómico y cultural de los estudiantes de la carrera de odontología de la universidad nacional de Loja y su relación con el estado de salud buco-dental, en el periodo octubre - marzo 2018. 
Nivel socioeconómico y cultural de los estudiantes de la carrera de odontología de la Universidad Nacional de Loja y su relación con el estado de salud buco-dental

El universo estuvo conformado por 270 estudiantes, la muestra fue de 230 estudiantes de acuerdo a los criterios de inclusión de la investigación.

Sobre la base de las variables establecidas internacionalmente se incluyeron las siguientes:

$\checkmark$ Índice de CPO-D: Resulta de la sumatoria de dientes permanentes cariados, perdidos y obturados, dividido para el total de la población examinada.

Los resultados obtenidos se valoraron mediante los siguientes rangos:

- De 0 a 1.1: Muy bajo

- De 1.2 a 2.6: bajo

- De 2,7 a 4.4: Moderado

- De 4.5 a 6.5: Alto

$\checkmark$ Índice de higiene oral simplificado: El IHO -S utiliza seis superficies dentarias que representan todos los segmentos posteriores y anteriores de la boca. Con este índice se mide la superficie del diente cubierta por restos y cálculo. El IHO-S se los obtiene de la sumatoria del índice de restos o placa bacteriana simplificado (IR-S) y el índice de cálculo simplificado (IC-S), dividido para 2

IHO- S= (IR- S+IC- S)/2

Los resultados obtenidos se valoraron bajo los siguientes rangos:

- Bueno: 0-1.2

- Regular: 1.3

- Malo: 3.1-6 
Deisy P. Saraguro-Ortega; Diego R. Aguilar-Ochoa; Jhoanna A. Riofrio-Herrera; Tannya L. Valarezo-Bravo; Ana M.

\section{$\checkmark$ Índice de Necesidad de Tratamiento Periodontal}

En el examen físico se empleó espejo plano bucal № 5 y sonda periodontal 621, recomendada por la OMS, la cual presenta una punta redondeada de $0,5 \mathrm{~mm}$ de diámetro y un área coloreada de 3,5 hasta 5,5 mm, para medir la profundidad de la bolsa y detectar cálculo subgingival.

Se tuvieron en cuenta los siguientes indicadores del estado periodontal en orden descendente de gravedad:

- Código X: excluido (1 o ningún diente presente en el sextante)

- Código 4: bolsas periodontales profundas (6 mm o más)

- Código 3: bolsas periodontales poco profundas (4-5 $\mathrm{mm})$

- Código 2: presencia de cálculo, caries, u otro factor retentivo de placa

- Código 1: presencia de sangramiento gingival al sondeo

- Código 0: tejido sano

\section{Clasificación de las necesidades de tratamiento:}

- NT 0: En caso de salud gingival

- NT 1: Necesidad de mejorar la higiene bucal si se ha registrado un código de1

- NT 2: Necesidad de raspado radicular, de eliminación de restauraciones desbordantes y de mejorar la higiene bucal (códigos 2+3)

- NT 3: Tratamiento complejo (código). 
Nivel socioeconómico y cultural de los estudiantes de la carrera de odontología de la Universidad Nacional de Loja y su relación con el estado de salud buco-dental

\section{$\checkmark$ Índice de Enfermedad Periodontal (IEP) de Ramfjord}

Los resultados obtenidos se valoraron bajo los siguientes rangos:

- 0: Ausencia de inflamación

- 1: Cambios inflamatorios leves a moderados que no, se extienden alrededor de todo el diente.

- 2: Gingivitis leve a moderada grave que se extiende alrededor de todo el diente.

- 3: Gingivitis intensa que se caracteriza por color rojo intenso, tendencia a la hemorragia y ulceración. (González \& Montero, 2013)

\section{Los instrumentos usados fueron:}

Encuesta: fue aplicada a los estudiantes, para evaluar el nivel socioeconómico se utilizó la encuesta aplicada por el INEC, conto de 25 preguntas en las cuales se evalúan las dimensiones de vivienda, educación, económica, bienes, tecnología y hábitos de consumo, cada una de las preguntas presentaban diversos puntajes y fueron establecidos según la respuesta de cada estudiante. Para poder evaluar el nivel cultural se aplicó una encuesta que consta de dos partes una: que determinaba las actitudes y la otra las creencias en salud oral de cada estudiante objeto de estudio.

\section{Se realizó la historia clínica odontológica}

Se analizaron los datos obtenidos: la historia clínica, la ficha socioeconómica y la encuesta cultural. Se representaron los resultados en tablas para su respectivo análisis, además de que se les aplico la prueba C hi cuadrado de Pearson en el caso del nivel socioeconómico y ANOVA para lo cultural, como medida de resumen se utilizó en por ciento. 
Deisy P. Saraguro-Ortega; Diego R. Aguilar-Ochoa; Jhoanna A. Riofrio-Herrera; Tannya L. Valarezo-Bravo; Ana M.

\section{Resultados.}

La tabla 1 muestra que de los 230 estudiantes en los cuales se realizó el estudio el $21.30 \%$ corresponde a una clase alta, $42,1 \%$ pertenecen a una clase medio alto, $28,26 \%$ corresponde a una clase medio típico; $8,26 \%$ pertenecen a un nivel medio bajo.

Tabla 1 Nivel socioeconómico de los estudiantes de la carrera de odontología, año 2018

\begin{tabular}{|l|c|c|}
\hline & Frecuencia & $\mathbf{\%}$ \\
\hline Alto (A) & 49 & 21.30 \\
\hline Medio alto (B) & 97 & 42.1 \\
\hline Medio típico (C) & 65 & 28.26 \\
\hline Medio bajo (C -) & 19 & 8.26 \\
\hline Total & 230 & 100 \\
\hline
\end{tabular}

Al analizar la tabla 2 se halló los siguientes resultados, el $91.0 \%$ que corresponde a 209 estudiantes indican tener un buen nivel cultural, mientras que el $9.0 \%$ correspondiente al $9 \%$ presenta un nivel cultural mal.

Tabla 2 Nivel cultural de los estudiantes de la carrera de odontología

\begin{tabular}{|c|c|c|}
\hline Nivel cultural & Frecuencia & $\mathbf{\%}$ \\
\hline Bueno & 209 & 91.0 \\
\hline Malo & 21 & 9.0 \\
\hline Total & 230 & 100 \\
\hline
\end{tabular}


Nivel socioeconómico y cultural de los estudiantes de la carrera de odontología de la Universidad Nacional de Loja y su relación con el estado de salud buco-dental

Como se observa en la tabla 3 de los 230 estudiantes que fueron examinados se pudo observar que el $54,35 \%$ presento enfermedad periodontal, 46,09\% presento hábitos para funcionales, $26,96 \%$ se pudo evidenciar la presencia de mal posición dentaria, 16,96 \% presento caries.

Tabla 3 Patologías de mayor frecuencia de los estudiantes de la carrera de odontología

\begin{tabular}{|l|c|c|}
\hline Patologías de mayor frecuencia & Frecuencia & \% \\
\hline Caries & 39 & 16.96 \\
\hline Enfermedad periodontal & 125 & 54.35 \\
\hline Mal posición dentaria & 62 & 26.96 \\
\hline Hábitos para funcionales & 106 & 46.09 \\
\hline
\end{tabular}

En esta serie (tabla 4) de los 230 estudiantes de la carrera de odontología, 97 estudiantes presentan un nivel socioeconómico medio alto: de los cuales el 8,2\% presentan caries y el 91,8\% no lo presenta, 65 estudiantes presentan un nivel socioeconómico medio típico: de los cuales el 27,7\% presentan dicha patología y el 72,3\% no la presenta, 49 presentan un nivel socioeconómico Alto: de los cuales el 8,2\% presentan caries y el 91,8\% no lo presenta, finalmente 19 estudiantes son de nivel socioeconómico medio bajo: en los que el 47,4\% tienen caries dental, y el 52,6\% no la poseen. En la prueba Chi cuadrado de Pearson, el valor del nivel de significación (Sig. Asintótica (2 caras $)=0,000)$ es inferior a $0,05(95 \%$ de confiabilidad $)$, luego los porcentajes de los niveles socio - económicos no son similares en el Índice de CPO - D. (Existe influencia) 
Deisy P. Saraguro-Ortega; Diego R. Aguilar-Ochoa; Jhoanna A. Riofrio-Herrera; Tannya L. Valarezo-Bravo; Ana M. Granda-Loaiza

Tabla 4 Caries * Nivel Socio-económico

\begin{tabular}{|c|c|c|c|c|c|c|c|}
\hline \multicolumn{8}{|c|}{ Caries. nivel socio económico } \\
\hline & & & \multicolumn{5}{|c|}{ Nivel socio- económico } \\
\hline & & & Alto & $\begin{array}{c}\text { Medio } \\
\text { alto }\end{array}$ & $\begin{array}{l}\text { Medio } \\
\text { típico }\end{array}$ & $\begin{array}{c}\text { Medio } \\
\text { bajo }\end{array}$ & Total \\
\hline \multirow[t]{4}{*}{ Caries } & \multirow[t]{2}{*}{ Presenta } & Frecuencia & 4 & 8 & 18 & 9 & 39 \\
\hline & & $\%$ & 8.2 & 8.2 & 27.7 & 47.7 & 17.0 \\
\hline & \multirow[t]{2}{*}{ No presenta } & Frecuencia & 45 & 89 & 47 & 10 & 191 \\
\hline & & $\%$ & 91.8 & 91.8 & 72.3 & 52.6 & 83.0 \\
\hline \multirow[t]{2}{*}{ Total } & & Frecuencia & 49 & 97 & 65 & 19 & 230 \\
\hline & & $\%$ & 100.0 & 100.0 & 100.0 & 100.0 & 100.0 \\
\hline
\end{tabular}

En cuanto a la mal posición dentaria y el nivel socio- económico 97 estudiantes presentan un nivel socioeconómico medio alto: de los cuales el 77,3\% no tienen mal posición dentaria, y el 22,7\% tienen mal posición; 65 estudiantes presentan un nivel socioeconómico medio típico: de los cuales el $63,1 \%$ no presentan dicha patología y el 36,9\% si la presenta, 49 presentan un nivel socioeconómico Alto: de los cuales el $83,7 \%$ no presentan mal posición dentaria y el 16,3\% si lo 
Nivel socioeconómico y cultural de los estudiantes de la carrera de odontología de la Universidad Nacional de Loja y su relación con el estado de salud buco-dental

presenta; finalmente 19 estudiantes son de nivel socioeconómico medio bajo: en los que el 57,9\% no tienen mal posición, y el 42,1\% tienen mala posición dentaria. (tabla 5 ). En la prueba Chi cuadrado de Pearson, el valor del nivel de significación (Sig. asintótica $(2$ caras) $=0,027)$ es inferior a 0,05 (95\% de confiabilidad), luego los porcentajes de los niveles socio -económicos no son similares en la Mala posición dentaria. (Existe influencia).

Tabla 5 Cruzadas: Mal posición dentaria * Nivel Socio-económico

\begin{tabular}{|c|c|c|c|c|c|c|c|}
\hline \multicolumn{8}{|c|}{ Mal posición dentaria * Nivel Socio-económico } \\
\hline & & & \multicolumn{5}{|c|}{ Nivel socio- económico } \\
\hline & & & Alto & $\begin{array}{c}\text { Medio } \\
\text { alto }\end{array}$ & $\begin{array}{l}\text { Medio } \\
\text { típico }\end{array}$ & $\begin{array}{c}\text { Medio } \\
\text { bajo }\end{array}$ & Total \\
\hline \multirow{4}{*}{$\begin{array}{l}\text { Mal } \\
\text { posición } \\
\text { dentaria }\end{array}$} & Presenta & Frecuencia & 41 & 75 & 41 & 11 & 168 \\
\hline & & $\%$ & 83.7 & 77.3 & 63.1 & 57.9 & 73.0 \\
\hline & No presenta & Frecuencia & 8 & 22 & 24 & 8 & 62 \\
\hline & & $\%$ & 16.3 & 22.7 & 36.9 & 42.1 & 27.0 \\
\hline Total & & Frecuencia & 49 & 97 & 65 & 19 & 230 \\
\hline
\end{tabular}


Deisy P. Saraguro-Ortega; Diego R. Aguilar-Ochoa; Jhoanna A. Riofrio-Herrera; Tannya L. Valarezo-Bravo; Ana M. Granda-Loaiza

\begin{tabular}{|l|l|l|l|l|l|l|l|}
\hline & $\%$ & 100.0 & 100.0 & 100.0 & 100.0 & 100.0 \\
\hline
\end{tabular}

Obsérvese en la tabla 6,49 estudiantes presentan un nivel socioeconómico Alto: de los cuales el $61,2 \%$ no presentan hábitos para funcionales y el $38,8 \%$ si lo presenta; 97 estudiantes presentan un nivel socioeconómico medio alto: de los cuales el 50,5\% no tienen hábitos para funcionales, y el 49,5\% tienen este tipo de hábitos; mientras que 65 estudiantes presentan un nivel socioeconómico medio típico: de los cuales el 55,4\% no presentan dicha patología y el 44,6 \% si la presenta; y, finalmente 19 estudiantes son de nivel socioeconómico medio bajo: en los que el 42,1\% no tienen estos hábitos, y el 57,9 \% tienen dichos hábitos. En la prueba Chi cuadrado de Pearson, el valor del nivel de significación (Sig. asintótica $(2$ caras $)=0,456)$ es superior a 0,05 (95\% de confiabilidad), luego los porcentajes de los niveles socioeconómicos son similares en los hábitos para funcionales. (No existe influencia).

Tabla 6 Hábitos para funcionales * Nivel Socio - económico

\begin{tabular}{|c|c|c|c|c|c|c|c|}
\hline \multicolumn{8}{|c|}{ Hábitos parafuncionales * Nivel Socio - económico } \\
\hline & & & \multicolumn{5}{|c|}{ Nivel socio- económico } \\
\hline & & & Alto & $\begin{array}{c}\text { Medio } \\
\text { alto }\end{array}$ & $\begin{array}{l}\text { Medio } \\
\text { típico }\end{array}$ & $\begin{array}{c}\text { Medio } \\
\text { bajo }\end{array}$ & Total \\
\hline Hábitos & Presenta & Frecuencia & 30 & 49 & 36 & 8 & 123 \\
\hline
\end{tabular}


Nivel socioeconómico y cultural de los estudiantes de la carrera de odontología de la Universidad Nacional de Loja y su relación con el estado de salud buco-dental

\begin{tabular}{|c|c|c|c|c|c|c|c|}
\hline \multirow{3}{*}{$\begin{array}{l}\text { para } \\
\text { funcionales }\end{array}$} & & $\%$ & 61.2 & 50.5 & 55.4 & 42.1 & 53.5 \\
\hline & \multirow[t]{2}{*}{ No presenta } & Frecuencia & 19 & 48 & 29 & 11 & 107 \\
\hline & & $\%$ & 38.8 & 49.5 & 44.6 & 57.9 & 46.5 \\
\hline \multirow[t]{2}{*}{ Total } & & Frecuencia & 49 & 97 & 65 & 19 & 230 \\
\hline & & $\%$ & 100.0 & 100.0 & 100.0 & 100.0 & 100.0 \\
\hline
\end{tabular}

Al estudiar el índice de Necesidad de Tratamiento Periodontal PNTC * Nivel Socio - económico los resultados evidenciaran que los estudiantes de nivel Alto: el 65,3\% tienen código 0, el 26,5\% tiene código 1 y el 8,2\% tiene código 2; los estudiantes de nivel Medio alto: el 49,5\% tienen código 0, el 40,2\% tiene código 1 y el 10,3\% tiene código 2; y los estudiantes de nivel Medio típico: el 27,7\% tienen código 0 , el 53,8\% tiene código 1 y el 18,5\% tiene código 2; finalmente los de nivel Medio bajo: el 36,8\% tienen código 0 , el 26,3\% tiene código 1 y el 36,8\% tiene código 2. Donde En la prueba Chi cuadrado de Pearson, el valor del nivel de significación (Sig. asintótica (2 caras) $=$ $0,000)$ es inferior a 0,05 ( $95 \%$ de confiabilidad), luego los porcentajes de los niveles socio económicos no son similares en el Índice PSR. (Existe influencia).

En cuanto a la relación Índice periodontal de Ramfjord * Nivel Socio - Económico los estudiantes que corresponden a un nivel Alto: el 63,3\% tienen código 0, el 28,6\% tiene código 1 y el 8,2\% tiene código 2, los correspondientes al nivel Medio alto: el 62,9\% tienen código 0, el 26,8\% tiene código 1 y el 10,3\% tiene código 2 mientras que los estudiantes de nivel socioeconómico Medio típico: el 12,3\% tienen código 0 , el 52,3\% tiene código 1 y el 35,4\% tiene código 2 ; y los estudiantes de nivel 
Deisy P. Saraguro-Ortega; Diego R. Aguilar-Ochoa; Jhoanna A. Riofrio-Herrera; Tannya L. Valarezo-Bravo; Ana M.

Medio bajo: el 26,3\% tienen código 0, el 26,3\% tiene código 1 y el 47,4\% tiene código 2. En la prueba Chi cuadrado de Pearson, el valor del nivel de significación (Sig. asintótica $(2$ caras) $=$ $0,000)$ es inferior a $0,05(95 \%$ de confiabilidad), luego los porcentajes de los niveles socio económicos no son similares en el Índice periodontal de Ramfjord. (Existe influencia).

Los resultados que se obtuvieron al relacionar la variable cultura con el índice de caries dental, mal posición dentaria, hábitos parafuncionales, necesidad de tratamiento parodontal y en el Índice periodontal de Ramfjord demostró con la prueba ANOVA, que no existen diferencias significativas en lo cultural entre si presentan.

\section{Discusión.}

La salud bucodental al igual que el concepto de salud general, también precisa de la asociación con los factores socioeconómicos, ambientales, y estilos de vida, y es necesario verla de una manera holística en donde intervienen tanto de forma positiva como negativa estos elementos y no solo como la ausencia de enfermedad. Dentro de la concepción social de la salud revisten gran importancia los determinantes sociales de la salud (DSS) en la población; por lo tanto el ejercicio de la Salud pública es una pieza clave para ofrecer mejores medidas de atención, la OMS establece que salud pública son las acciones colectivas e individuales del estado y la sociedad civil, dirigidas a mejorar la salud de la población. (Villa Ocampo, 2015)

La salud, desde el punto de vista singular y general, constituye un medio para la realización personal y colectiva, por ello, en la medida en que la sociedad tiene más desarrollo y es capaz de aportar más bienes y disfrutar de ellos con mayor homogeneidad, se dice que la situación de salud mejora. La situación de salud representa el conjunto de problemas de salud de grupos de población y formaciones sociales, y expresa sus formas de vida cotidiana, en sus dimensiones: general (el modo 
Nivel socioeconómico y cultural de los estudiantes de la carrera de odontología de la Universidad Nacional de Loja y su relación con el estado de salud buco-dental

de vida), particular (condiciones de vida) y singular (el estilo de vida). (Martínez, Capote, Bermúdez, \& Martínez, 2014)

La carga de enfermedades bucodentales es más alta en los grupos sociales más desfavorecidos y las influencias sociales, económicas, comportamentales y ambientales tienen gran importancia ya que van a definir las pautas de morbilidad bucodental en las distintas áreas geográficas que serán un reflejo de los diferentes perfiles de riesgo y la efectividad de los programas preventivos de atención bucodental.

En el orden de los razonamientos anteriores en la odontología las diferencias que existen entre el nivel socioeconómico genera tratamientos diferentes, por lo tanto no existe una postura ética sobre las necesidades en salud bucal de la población que no puede pagar; entonces la odontología se convierte al parecer en un bien de consumo, en donde se ofrece prevención a los pobres y tratamientos a los ricos; sin embargo no solo existen barreras económicas, también hay barreras culturales como las que se reflejan en las diferentes percepciones del proceso salud - enfermedad. (Botello, Espinosa, \& Castroll, 2011)

La caries dental y la enfermedad periodontal son consideradas como los eventos de mayor peso en la historia de la morbilidad bucal a nivel mundial (Zacca, Sosa, \& Mojaiber, 2001). Estas son causadas por la presencia de una biopelícula producto de la organización estructural de diferentes tipos bacterianos dentro de un hábitat propicio, facilitado por la deficiente higiene bucal, que por sus mecanismos patológicos y los múltiples factores involucrados afectan a todos los individuos.

Su distribución y severidad varían de una región a otra y su aparición está fuertemente asociada con factores socioculturales, económicos, del ambiente y del comportamiento. Estudios realizado por Arrieta Vergara K. 2011 resulto que los estudiantes de odontología presentan altos porcentajes de 
Deisy P. Saraguro-Ortega; Diego R. Aguilar-Ochoa; Jhoanna A. Riofrio-Herrera; Tannya L. Valarezo-Bravo; Ana M.

caries y enfermedad periodontal. (Arrieta, Díaz, \& González, 2011), (Socransky \& Haffajee, 2002), (Pérez, 2005)

Es medular expresar que para conseguir que la Enfermedad Periodontal se trate como una verdadera enfermedad inflamatoria crónica, es necesario identificar el contexto actual y plantear una visión estratégica que permita entre otras cuestiones, visualizar algunos problemas desde un contexto social, así como con los indicadores que propician y que de alguna u otra manera nos explican la morbilidad, como serían la pobreza, la educación, la nutrición y estilos de vida. (Villa Ocampo, 2015)

Los resultados obtenidos en la presente investigación coinciden con autores como: (González, González, \& Gónzalez, 2013), (Sanabria, Suárez, \& Estrada, 2015) quienes concluyen que el nivel socioeconómico si influye directamente en la salud bucal, pues en sus estudios realizados encontraron que las personas de un nivel socioeconómico alto presentaban índices de caries muy bajos, resultados que son similares a los evidenciados en la presente investigación: en los cuales se pudo determinar que aquella población que cuenta con un nivel socioeconómico alto presenta un índice de caries bajo, equivalente al $38,8 \%$ de la población de estudio; mientras que, los de nivel socioeconómico medio bajo, el índice de caries es alto en un $45,7 \%$, dichos resultados fueron comprobados mediante la prueba estadística del Chi cuadrado de Pearson, que obtuvo un nivel de significancia de 0,0 resultado inferior al margen de error planteado para este estudio, demostrando que el nivel socioeconómico si influye en el estado de salud bucodental. (González, González, \& Gónzalez, 2013), (Sanabria, Suárez, \& Estrada, 2015)

Sin embargo (Hidalgo Terán, 2015) al comparar las condiciones socioeconómicas de los alumnos de ciclo básico de la Unidad Educativa" Rincón del Saber", evidenció que a mejor 
Nivel socioeconómico y cultural de los estudiantes de la carrera de odontología de la Universidad Nacional de Loja y su relación con el estado de salud buco-dental

condición socioeconómica, mayor es el problema de salud oral, pues en su estudio encontró un índice de caries alto de 5,80\% en la población de nivel socioeconómico medio alto, resultados que se contraponen a los obtenidos en este trabajo. (Hidalgo Terán, 2015)

Igualmente en el estudio de Martinez, EM (Martinez Mejia, 2017) menciona, que la población presenta un nivel socioeconómico bueno y su índice CPOD es de 4,1\% considerado dentro de los rangos de niveles de severidad en prevalencia de caries según la OMS como moderado de tal manera que se muestran contrarios a los obtenidos en la presente investigación. (Martinez Mejia, 2017)

Finalmente se puede establecer de acuerdo a los estudios analizados que entre más edad de la población mayor es la experiencia de caries y enfermedad periodontal, la OMS reporta que el índice de caries a nivel mundial es de 2,5\% a la edad de 12 años, valor que se va incrementando a lo largo de la vida, tal es el caso del presente estudio al tratarse de estudiantes de una edad comprendida entre 19 a 26 años se evidencia un índice CPOD moderado, que al valorarlo por separado se puede observar que la prevalencia de caries es menor que la de dientes obturados, por lo tanto es importante mencionar que según (Espinoza Solano \& León-Manco, 2015) este indicador determina si el individuo ha sufrido caries a lo largo de su vida pero estos han debido tener una atención odontológica, lo cual demuestra la baja prevalencia pero la alta experiencia de caries dental. (Espinoza Solano \& León-Manco, 2015)

Para concluir al establecer la relación entre el nivel socioeconómico y cultural con las patologías de mayor frecuencia encontradas en este estudio se determinó que la caries, enfermedad periodontal y la mal posición dentaria tienen relación directa con la situación socioeconómica y cultural de los estudiantes con un nivel de significancia de 0,0 de la prueba estadística Chi cuadrado 
Deisy P. Saraguro-Ortega; Diego R. Aguilar-Ochoa; Jhoanna A. Riofrio-Herrera; Tannya L. Valarezo-Bravo; Ana M.

de Pearson. En cuanto a los hábitos para funcionales al obtener un nivel de significancia de 0,4 se concluyó que no existe influencia entre el nivel socioeconómico y cultural con el estado de salud bucodental.

\section{Bibliografia.}

Arrieta, K. M., Díaz, A., \& González, F. (2011). Prevalencia de caries y enfermedad periodontal en estudiantes de odontología. Revista Cubana de Estomatología, 48(1), 6-13.

Botello, N., Espinosa, A., \& Castroll, M. (2011). Prevalencia, severidad y extension de periodontitis crónica. Revista Odontológicas Mexicana, 15(1), 31-39.

Bravo, M., Casals, E., Cortes, F. J., \& Llodra, J. C. (2006). Encuesta de Salud oral en España 2005. RCOE, 11(4), 409-456.

Cuenca, E. (2005). Las desigualdades en salud oral. En E. Cuenca, \& P. Baca, Odontología preventiva y Comunitaria, principios, métods y aplicaciones (págs. 263-74). Barcelona: Masson.

Espinoza Solano, M., \& León-Manco, R. (2015). Prevalencia y experiencia de caries dental en estudiantes segun facultades de una universidad particular ecuatoriana. Rev Estomatol Herediana, 25(3), 187-193.

González, A., González, B., \& Gónzalez, E. (2013). Salud dental : relación entre la caires dental y el consumo de alimentos. Nutrición Hospitalaria, 28(4), 64-71.

González, G., \& Montero, M. (2013). Estomatología General Integral. La Habana: Ciencias Médicas .

Hidalgo Terán, S. (2015). Relación del estado de salud dental con factores socioeconómicos de los alumnos del ciclo básico de la unidad educativa rincón del saber periodo junio - julio 2014. Quito: Universidad Central del Ecuador.

Martinez Mejia, E. (2017). Prevalencia de caries y su relación con determinantes sociales de la salud en niños atendidos por estudiantes de la Facultad de Odontología, en el proyecto de vinculación con la comunidad en la Escuela "Padre Juan de Velasco", comunidad de Pucará. Quito: Universidad Central del Ecuador.

Martínez, J., Capote, J., Bermúdez, G., \& Martínez, Y. (2014). Determinantes sociales del estado de salud oral en el contexto actual. MediSur, 12(4), 562-569.

Pérez, A. (2005). La Biopelícula: Una nueva visión de la placa dental. Rev Estomatol Herediana, $5(1), 82-85$.

Petersen, P. E., Bourgeois, D., Ogawa, H., Estupinan-Day, S., \& Ndiaye, C. (2005). The global burden of oral diseases and risks to oral health. Bull World Health Organ, 83(9), 661-9. 
Nivel socioeconómico y cultural de los estudiantes de la carrera de odontología de la Universidad Nacional de Loja y su relación con el estado de salud buco-dental

Sanabria, C., Suárez, M., \& Estrada, J. (2015). Relación entre determinantes socioeconómicos, cobertura en salud y caires dental en veinte países. Rev Gerenc Polit .Salud, 14(28), 161-189. Obtenido de http://dx.doi.org/10.11144/Javeriana.rgyps 18-28.rdsc

Socransky, S., \& Haffajee, A. (2002). Dental biofilms: difficult therapeutic targets. Periodontol 2000, 28(1), 12-55.

Villa Ocampo, P. (2015). Enfoque salubrista de la enfermedad periodontal. Revista Iberoamericana de Ciencias, 2(4), 179-189.

Zacca, G., Sosa, M., \& Mojaiber, A. (2001). Situación de salud bucal de la población cubana. Estudio comparativo según provincias, 1998. Revista Cubana de Estomatología, 39(2), 90110. 